\title{
Early Effects of Prolonged Cardiac Arrest and Ischemic Postconditioning during Cardiopulmonary Resuscitation on Cardiac and Brain Mitochondrial Function in Pigs
}

\author{
Timothy R. Matsuura, BA ${ }^{1}$, Jason A. Bartos, $\mathrm{MD}, \mathrm{PhD}^{2}$, Adamantios Tsangaris, $\mathrm{MD}^{2}$, \\ Kadambari Chandra Shekar, MS ${ }^{1}$, Matthew D. Olson, MD ${ }^{2}$, Matthias L. Riess, MD, PhD $^{3,4,5}$, \\ Martin Bienengraeber, $\mathrm{PhD}^{6}$, Tom P. Aufderheide, MD, MS ${ }^{7}$, Robert W. Neumar, MD, $\mathrm{PhD}^{8}$, \\ Jennifer N. Rees, PhD $^{2}$, Scott H. McKnite, BS ${ }^{2}$, Anna E. Dikalova, PhD $^{5}$, Sergey I. Dikalov, \\ $\mathrm{PhD}^{5}$, Hunter F. Douglas, $\mathbf{B S}^{4}$, and Demetris Yannopoulos, $\mathbf{M D}^{2}$ \\ ${ }^{1}$ Department of Integrative Biology and Physiology, University of Minnesota, Minneapolis, MN \\ 2Department of Medicine-Cardiovascular Division, University of Minnesota, Minneapolis, MN \\ ${ }^{3}$ Department of Anesthesiology, TVHS VA Medical Center, Nashville, TN \\ ${ }^{4}$ Department of Anesthesiology Vanderbilt University Medical Center, Nashville, TN \\ ${ }^{5}$ Department of Pharmacology Vanderbilt University Medical Center, Nashville, TN \\ ${ }^{6}$ Departments of Anesthesiology and Pharmacology \& Toxicology, Medical College of Wisconsin, \\ Milwaukee, Wisconsin \\ ${ }^{7}$ Department of Emergency Medicine, Medical College of Wisconsin, Milwaukee, Wisconsin \\ ${ }^{8}$ Department of Emergency Medicine, University of Michigan, Ann Arbor, Michigan
}

\section{Abstract}

Background-Out-of-hospital cardiac arrest (CA) is a prevalent medical crisis resulting in severe injury to the heart and brain and an overall survival of less than 10 percent. Mitochondrial dysfunction is predicted to be a key determinant of poor outcomes following prolonged CA. However, the onset and severity of mitochondrial dysfunction during CA and cardiopulmonary resuscitation (CPR) is not fully understood. Ischemic postconditioning (IPC), controlled pauses during the initiation of CPR, has been shown to improve cardiac function and neurologically favorable outcomes after fifteen minutes of $\mathrm{CA}$. We tested the hypothesis that mitochondrial dysfunction develops during prolonged CA and can be rescued with IPC during CPR (IPC-CPR).

\begin{abstract}
Methods-63 swine were randomized to no ischemia (Naïve), nineteen minutes of ventricular fibrillation (VF) CA without CPR (Untreated VF), or fifteen minutes of CA with 4 minutes of reperfusion with either standard CPR (S-CPR) or IPC-CPR. Mitochondria were isolated from the
\end{abstract}

Corresponding Author: Demetris Yannopoulos, UMN - Cardiology Division, 420 Delaware SE, Minneapolis, MN 55455, Telephone Number: 612-626-1382, Fax Number: 612-626-4411, yanno001@umn.edu.

Publisher's Disclaimer: This is a PDF file of an unedited manuscript that has been accepted for publication. As a service to our customers we are providing this early version of the manuscript. The manuscript will undergo copyediting, typesetting, and review of the resulting proof before it is published in its final citable form. Please note that during the production process errors may be discovered which could affect the content, and all legal disclaimers that apply to the journal pertain. 
heart and brain to quantify respiration, rate of ATP synthesis, and calcium retention capacity (CRC). Reactive oxygen species (ROS) production was quantified from fresh frozen heart and brain tissue.

Results-Compared to Naïve, Untreated VF induced cardiac and brain ROS overproduction concurrent with decreased mitochondrial respiratory coupling and CRC, as well as decreased cardiac ATP synthesis. Compared to VF CA, S-CPR attenuated brain ROS overproduction but had no other effect on mitochondrial function in the heart or brain. Compared to VF CA, IPC-CPR improved cardiac mitochondrial respiratory coupling and rate of ATP synthesis, and decreased ROS overproduction in the heart and brain.

Conclusions-Fifteen minutes of VF CA results in diminished mitochondrial respiration, ATP synthesis, CRC, and increased ROS production in the heart and brain. IPC-CPR attenuates cardiac mitochondrial dysfunction caused by prolonged VF CA after only 4 minutes of reperfusion, suggesting that IPC-CPR is an effective intervention to reduce cardiac injury. However, reperfusion with both CPR methods had limited effect on mitochondrial function in the brain, emphasizing an important physiological divergence in post-arrest recovery between those two vital organs.

\section{Graphical abstract}

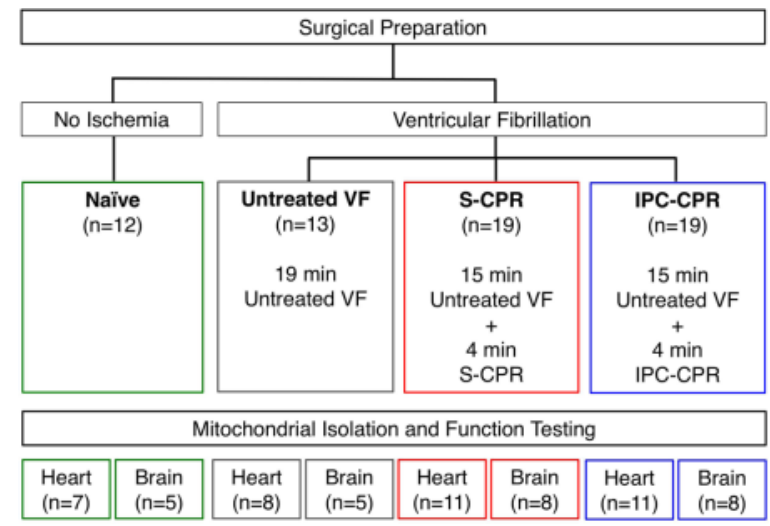

\section{Keywords}

cardiac arrest; ventricular fibrillation; cardiopulmonary resuscitation; mitochondria; ischemic postconditioning; reperfusion injury

\section{INTRODUCTION}

Out-of-hospital cardiac arrest (CA) afflicts 395,000 people each year in the United States(1). On average, less than 6 percent survive, resulting in more than 360,000 deaths per year and an enormous public health burden(2). In the past five decades, improvements to cardiopulmonary resuscitation (CPR) and systems-based interventions have resulted in only modest improvements to outcomes following prolonged CA. Novel resuscitation strategies are necessary to further improve survival. 
One such novel strategy is ischemic postconditioning (IPC), a therapy delivered upon reperfusion after prolonged ischemia to mitigate cellular injury caused by ischemia and reperfusion (IR)(3,4). IPC is accomplished using several brief interruptions in blood flow at the onset of reperfusion. Our laboratory applied IPC during CPR in a porcine model of prolonged whole-body ischemia during ventricular fibrillation (VF) CA. Pauses in chest compressions during the first 2 minutes of CPR improved left ventricular ejection fraction (LVEF) following return of spontaneous circulation (ROSC) and increased neurologically favorable survival after 48 hours(5-7). These data demonstrate that the method of initial reperfusion with CPR has an impact on the extent of injury after prolonged cardiac arrest.

Mitochondria are implicated in the pathophysiology of IR injury and also provide a nexus for integrating the protective molecular pathways activated by IPC $(8-10)$. Therefore, we sought to investigate the effect that VF, CPR, and IPC have on mitochondrial function in the heart and brain. We hypothesized that mitochondrial function: 1) is significantly depressed after prolonged cardiac arrest, and 2) can be improved with IPC at the initiation of CPR (IPC-CPR). Mitochondrial responses to ischemia, CPR, and IPC were characterized without attempting defibrillations to eliminate confounders such as success and timing of ROSC, refractory VF, number of defibrillations, antiarrhythmics, and vasopressor support. Understanding the early physiological consequences of the key components of cardiac arrest and resuscitation will have significant impact on the direction and refinement of future therapies.

\section{METHODS}

All studies were performed with approval from the Institutional Animal Care and Use Committee of the Minneapolis Medical Research Foundation and the University of Minnesota in accordance with the National Research Council's Guidelines for the Care and Use of Laboratory Animals(11). Animal preparation has been described previously(6). See Supplement for full description of methods.

\section{Experimental Protocols}

Following surgical preparation and baseline measurements, animals were randomized to one of 4 groups (Naïve, Untreated VF, S-CPR, and IPC-CPR) and further to either heart or brain isolation (Fig 1). Twelve animals received no ischemia (Naïve, heart isolation [ $\mathrm{n}=7]$, brain isolation [ $\mathrm{n}=5])$. In the 3 ischemic groups, VF was induced via a pacing wire positioned in the right ventricle. During VF, ventilation and temperature management were discontinued. Mitochondria were isolated $19 \mathrm{~min}$ after initiation of VF in all 3 ischemic groups. To establish the effect of prolonged untreated VF CA, 13 animals were randomized to receive 19 min of VF CA without CPR (Untreated VF, heart [n = 8], brain [n=5]). To establish the effect of reperfusion by type of CPR on mitochondrial function, 38 animals were randomized to receive $15 \mathrm{~min}$ of untreated VF arrest followed by either 4 min of standard CPR (S-CPR, heart [ $\mathrm{n}=11]$, brain [ $\mathrm{n}=8]$ ) or 4 min of IPC-CPR (heart [ $=11]$, brain $[\mathrm{n}=$ $8])$. Epinephrine $(0.125 \mu \mathrm{g} / \mathrm{kg})$ was administered after the $3^{\text {rd }}$ minute of CPR. In the IPCCPR group, IPC was administered via 3 cycles of 20 seconds compressions/ventilations followed by 20 seconds pause in compressions/ventilations during the first 2 minutes of 
CPR. Both CPR groups received asynchronous ventilations at 10 breaths $/ \mathrm{min}$ and $10 \mathrm{ml} / \mathrm{kg}$, and 100 chest compressions/min with a target depth of $20 \%$ AP diameter and $50 \%$ duty cycle. Compressions were performed with an automated, custom-built, CPR piston device (Caztek Engineering, St. Paul, MN). Coronary perfusion pressure (CPP), the gradient between aortic and right atrial blood pressures during chest decompression, was calculated as an approximation of coronary blood flow and a measure of CPR quality. At the end of the $4^{\text {th }}$ minute of CPR, tissue was sampled for mitochondria isolation. No defibrillations were attempted.

At the conclusion of the experimental protocol, mitochondria were isolated at $4{ }^{\circ} \mathrm{C}$ via differential centrifugation from the left ventricle of the heart and brain as described previously(12,13). State 3 (S3) and State 4 (S4) respiration, the rate of ATP synthesis, and calcium retention capacity (CRC) were quantified as previously described(12). The respiratory control index (RCI) was calculated as the ratio of S3 to S4 respiration. Production of reactive oxygen species (ROS) was assessed with electron spin resonance (ESR) fresh frozen heart and brain tissue.

\section{Statistics}

Values are expressed as mean \pm standard error of the mean. ANOVA with Newman-Keuls posthoc test was used for comparisons between treatments. Unpaired two-tailed t-tests were used to compare hemodynamics during CPR between S-CPR and IPC-CPR. The nullhypothesis was rejected for $\mathrm{p}<0.05$.

\section{RESULTS}

Baseline hemodynamic parameters did not differ between Naïve, Untreated VF, S-CPR, or IPC-CPR groups (Table 1). As expected, pauses in chest compressions during IPC-CPR resulted in a lower average CPP during each pause compared to S-CPR (Fig 2a). However, IPC-CPR resulted in a higher CPP during the 4th minute of CPR after administration of epinephrine. Average CPP during CPR did not differ between S-CPR and IPC-CPR (Fig 2b).

\section{Mitochondrial Function}

Mitochondria were isolated from the heart and brain to quantify S3 and S4 respiration, RCI, rate of ATP synthesis, and CRC. ROS production was quantified in fresh frozen heart and brain tissue.

\section{I) Prolonged Ischemia (Untreated VF)}

a. Heart: Compared to Naïve, Untreated VF resulted in a significant decrease in RCI with both complex I and complex II substrates (Fig 3a,b). The decrease in RCI was predominantly driven by a rise in S4 respiration indicating uncoupling of oxidative phosphorylation (Fig 3e,f). The rate of ATP synthesis after Untreated VF was less than 50\% of Naïve hearts, consistent with the respiration profile described above (Fig 3g,h). Untreated VF resulted in decreased CRC with complex II substrates compared to Naïve (Figure 3j) and caused a significant increase in ROS production compared to Naïve hearts (Fig 5a). 
b. Brain: Compared to Naïve, Untreated VF significantly decreased RCI for complex II substrates (Fig 4b). Decreased complex II RCI was predominantly driven by a substantial decrease in S3 respiration with no change in S4 respiration (Fig 4d,f), whereas complex I stimulated S3 and S4 respiration decreased proportionally (Fig 4c,e). The rate of ATP synthesis was unaffected by Untreated VF compared to Naïve (Fig 4g,h). Untreated VF resulted in decreased CRC with both complex I and complex II substrates compared to Naïve (Fig 4i,j). ROS production significantly increased after Untreated VF compared to Naïve brain (Fig 5b).

\section{II) Reperfusion with S-CPR}

a. Heart: Compared to Untreated VF, 4 minutes of reperfusion with S-CPR lead to a progressive increase of RCI, particularly for complex II substrates, predominantly driven by increased S3 respiration while S4 respiration remained unchanged (Fig 3a-f). However, RCI remained decreased after reperfusion with S-CPR compared to Naïve due primarily to increased S4 respiration. S-CPR resulted in a non-significant increase in the rate of ATP synthesis compared to Untreated VF (Fig 3g,h). S-CPR did not affect CRC compared to Untreated VF (Fig 3i,j). ROS production in the myocardium was similar between S-CPR and Untreated VF and remained elevated compared to Naïve (Fig 5a).

b. Brain: Four minutes of reperfusion with S-CPR did not alter mitochondrial respiration, ATP synthesis, or CRC compared to Untreated VF (Fig 4). S-CPR significantly decreased ROS overproduction in the brain compared to Untreated VF to a level that remained elevated compared to Naïve treatment (Fig 5b).

\section{III) Reperfusion with IPC-CPR}

a. Heart: Compared to Untreated VF and S-CPR, 4 minutes of reperfusion with IPC significantly increased RCI for both complexes I and II to values similar to Naïve (Fig 3a,b). Increased RCI was a result of significantly decreased S4 respiration combined with nonsignificant increased S3 respiration, indicating tighter coupling of oxidative phosphorylation (Fig 3c-f). The rate of ATP synthesis normalized to the level of Naïve hearts and was significantly increased compared to Untreated VF (Fig 3g,h). IPC-CPR significantly improved CRC with complex I substrates compared to Untreated VF (Fig 3i) and decreased ROS production in the myocardium compared to both Untreated VF and S-CPR (Fig 5a).

b. Brain: Four minutes of reperfusion with IPC-CPR did not alter mitochondrial respiration, ATP synthesis, or CRC compared to Untreated VF (Fig 4). IPC-CPR significantly decreased ROS production in the brain compared to Untreated VF and S-CPR to a level that remained elevated compared to Naïve treatment (Fig 5b).

\section{DISCUSSION}

To our knowledge, this is the first study investigating the effect of IPC during high-quality $\mathrm{CPR}$ on mitochondrial function after prolonged $\mathrm{CA}$ in swine. To determine their role in the early pathogenesis and recovery after prolonged CA, mitochondrial responses to whole-body ischemia and to CPR reperfusion with and without IPC were characterized during ongoing 
CPR to eliminate the confounding impact of defibrillation attempts and independent of success or timing of ROSC. After 4 minutes of reperfusion, IPC-CPR attenuated mitochondrial dysfunction compared to Untreated VF and S-CPR. Untreated VF resulted in dysfunctional mitochondrial respiration, CRC, and ROS production in the heart and brain, as well as decreased cardiac mitochondrial ATP synthesis. S-CPR partially restored cardiac mitochondrial respiration and ATP synthesis, but was insufficient to decrease ROS overproduction. In contrast, IPC-CPR normalized cardiac mitochondrial respiration and ATP synthesis to non-ischemic levels. ROS overproduction in the brain decreased with S-CPR and was further attenuated with IPC-CPR, but brain mitochondrial function was otherwise unaffected by reperfusion with either method of CPR.

RCI quantifies the coupling of oxygen consumption to ATP synthesis during the primary mitochondrial function of oxidative phosphorylation. Untreated VF decreased RCI with both complex I and complex II substrates in cardiac mitochondria, consistent with findings that respiratory dysfunction impedes cardiac recovery after prolonged ischemia $(14,15)$. Compared to Untreated VF, reperfusion with S-CPR increased cardiac mitochondrial RCI with both complex I and complex II substrates. IPC-CPR further increased cardiac mitochondrial RCI compared to S-CPR, demonstrating a tighter coupling of oxygen consumption to ATP synthesis. The increase in cardiac mitochondrial RCI with IPC-CPR occurred in tandem with an increase in the rate of ATP synthesis. Maintenance of cardiac bioenergetics enhances the probability of successful resuscitation(16-18). Tighter coupling of oxidative phosphorylation with IPC-CPR may be critically important for efficient oxygen utilization during the hypoxic conditions of CA and hypoperfusion with CPR, and may thus minimize myocardial damage and preserve LVEF as previously observed $(5,6)$.

Increased intracellular and mitochondrial calcium concentrations promote open conformation of the mPTP(19). Early, prolonged opening of the mPTP during IR is proposed to be a fundamental determinant of cell death $(9,20)$. CRC quantifies the amount of calcium-mediated stress necessary to induce mPTP opening(21). Compared to Naïve animals, Untreated VF decreased CRC with complex II substrates in mitochondria isolated from the heart. Decreased CRC may be due to ROS overproduction $(22,23)$ or may reflect increased mitochondrial calcium levels in vivo during Untreated VF. Reperfusion with SCPR did not affect CRC, whereas IPC-CPR increased CRC with complex I substrates compared to Untreated VF. Decreased ROS overproduction or decreased in vivo calcium loading could contribute to improved calcium stress tolerance with IPC-CPR, though the identity of this mechanism remains unknown from these studies.

ESR intensity of oxidized aconitase is a marker of mitochondrial ROS production. Oxidized aconitase is inactive(24), potentiating a decreased flux through the TCA cycle. Untreated VF and S-CPR showed a significantly increased oxidized aconitase signal in the heart compared to Naïve. IPC-CPR dramatically reduced cardiac mitochondrial ROS production compared to Untreated VF and S-CPR. Attenuation of ROS overproduction improves cardiac contractility in isolated hearts(25) and could be a mechanism through which IPC improves LVEF after prolonged cardiac arrest $(5,6)$. 
In a previous study of cardiac and cerebral recovery in a porcine model of prolonged cardiac arrest, IPC-CPR was shown to improve post-resuscitation LVEF and decreased incidence of adverse events compared to S-CPR $(5,6)$. In the present study, cardiac mitochondrial respiration decreased dynamically during Untreated VF and S-CPR compared to Naïve and could be rescued early during resuscitation with IPC-CPR. Taken together, these data suggest a positive association between improved mitochondrial function during CPR and post-ROSC cardiac function. These data indicate that CPR is a therapeutic opportunity to modify the severity of cardiac IR injury in agreement with evidence from myocardial infarction that postconditioning therapies applied at the onset of reperfusion confer protection to the myocardium $(26,27)$. S-CPR alone also trended toward an increase in both $\mathrm{RCI}$ and rate of ATP synthesis compared to Untreated VF, corroborating the observation that perfusion with CPR improves probability of ROSC after prolonged cardiac arrest(28). Yeh et al. similarly observed preserved cardiac mitochondrial function and viability after reperfusion with CPR compared to time-controlled untreated CA(29). Thus, despite evidence of IR injury as demonstrated by the attenuation of mitochondrial dysfunction with IPC-CPR, establishing blood flow with CPR after prolonged CA should remain a primary goal of resuscitation.

In contrast to the reperfusion response of cardiac mitochondria, brain mitochondrial function declined during Untreated VF and did not respond within the first 4 minutes of reperfusion with S-CPR or IPC-CPR. S-CPR decreased ROS overproduction in the brain compared to Untreated VF, while IPC-CPR further decreased ROS overproduction compared to S-CPR. Untreated VF decreased RCI with complex II substrates and severely depressed CRC with both complex I and complex II substrates in mitochondria isolated from the brain. The rate of ATP synthesis was not significantly affected by treatment. Reperfusion with S-CPR had no effect on CRC in the heart or the brain compared to Untreated VF. The steep and irreversible decline in CRC may be indicative of increased sensitivity to MPTP opening specific to neuronal injury and calcium overload during IR(30-32).

In previous studies, IPC-CPR improved neurologically favorable survival while decreasing neuronal cell death after prolonged cardiac arrest $(5,6)$. IPC also reduced cell death and infarct size in models of cerebral ischemia $(33,34)$. In the present investigation, IPC-CPR did not alter cerebral mitochondrial function compared to Untreated VF or S-CPR early during resuscitation. This dissociation of early mitochondrial function and neurologic outcome may indicate that IPC-CPR does not confer increased neurologically favorable recovery via improvements in mitochondrial function during reperfusion with CPR. Alternatively, these findings may indicate that cerebral recovery does not occur as early as 4 minutes after the onset of reperfusion. In a recent study characterizing cerebral recovery from asphyxial arrest in swine, mitochondrial respiration remained depressed even after ROSC and 4 hours of recovery(35). Previous experiments demonstrated favorable neurologic outcomes after prolonged CA that continued to improve 24 hours post-ROSC, emphasizing a delayed period of neurologic recovery that may extend well beyond the duration of this investigation(5-7).

Heart and brain mitochondrial responses differed dramatically after reperfusion with CPR. Compared to Untreated VF, S-CPR improved coupling of mitochondrial respiration in the heart but had no effect in the brain. S-CPR decreased ROS overproduction compared to 
Untreated VF in the brain but not in the heart. IPC-CPR improved cardiac mitochondrial RCI and rate of ATP synthesis compared to Untreated VF and S-CPR, but its effect was absent in brain mitochondria. Thus, reperfusion with both CPR methods incrementally improved cardiac mitochondrial performance but had limited effect on mitochondrial function in the brain. This physiological divergence in acute post-arrest recovery may be critical for targeted treatment of these two vital organs.

CPP is an estimate of coronary blood flow. As expected, IPC-CPR decreased CPP during each 20-second pause compared to S-CPR. After completion of the controlled pauses and administration of epinephrine, IPC-CPR increased CPP during the 4th minute of CPR. A threshold perfusion pressure is hypothesized to be critical for predicting successful resuscitation(36). Thus, increased CPP in the $4^{\text {th }}$ minute may be a potential mechanism for improved cardiac mitochondrial function during IPC-CPR. Sensitivity to exogenous epinephrine may indicate that IPC-CPR protects vascular endothelium and smooth muscle. Coronary reflow and endothelial vasodilator response is attenuated after ischemia and reperfusion( 3,37$)$, and this response can be salvaged with IPC(38). During prolonged CA, increased revascularization and systemic vasoconstriction may be vital mechanisms through which IPC-CPR improves resuscitation success.

Several limitations to this study need to be acknowledged. The mechanism by which IPCCPR increased cardiac mitochondrial function was not determined. IPC-CPR increased perfusion pressure during the $4^{\text {th }}$ minute of CPR. Mitochondrial function may have improved as a direct result of obtaining a critical perfusion pressure threshold. Equivalent CPPs could not be generated in the control group to test the effect of blood flow on mitochondrial function. The high CPP obtained in both CPR groups and the lack of underlying coronary disease represent ideal CPR conditions that may limit the clinical translation of these outcomes. Intermittent compressions during IPC-CPR may contribute to variations in chest compliance and thus improve the mechanics of flow generation. The effect of IPC-CPR on thoracic compliance, endothelial function, and coronary flow will be the subject of future investigations. Additionally, the acute decrease in mitochondrial function described here, and its prevention by IPC-CPR, may be only a portion of the pathogenesis of prolonged cardiac arrest. Reperfusion injury can take hours or days to fully manifest. The current data were obtained only 4 minutes after the onset of reperfusion and prior to ROSC to eliminate confounders such as defibrillation attempts, arrhythmias, or refractory VF. Injury may continue to accrue beyond this time, thus contributing to the low incidences of neurologically favorable survival even in patients who experience a ROSC.

\section{CONCLUSIONS}

Prolonged untreated CA induced ROS overproduction concurrent with decreased mitochondrial respiratory coupling and CRC in the heart and the brain. Additionally, cardiac mitochondrial ATP synthesis was depressed. S-CPR improved coupling of cardiac mitochondrial respiration, and partially attenuated ROS overproduction in the brain. After 4 minutes of reperfusion, IPC-CPR improved cardiac mitochondrial function as indicated by increased respiratory coupling and rate of ATP synthesis, as well as decreased ROS overproduction. These results indicate potential mechanisms through which IPC-CPR 
improves cardiac performance following prolonged cardiac arrest. In contrast, IPC-CPR decreased ROS production but did not otherwise improve mitochondrial function in the brain, emphasizing an important physiological divergence in post-arrest recovery between these two vital organs.

\section{Supplementary Material}

Refer to Web version on PubMed Central for supplementary material.

\section{Acknowledgments}

The authors wish to thank Rachel Stark and Qunli Cheng, MD for their valuable contributions.

\section{SOURCES OF FUNDING}

This work was supported by the NIH R01HL123227 (DY) and the American Heart Association 16PRE27600038 (TRM).

\section{References}

1. Daya MR, Schmicker RH, Morrison LJ. Current burden of cardiac arrest in the United States: report from the resuscitation outcomes consortium. 2015

2. Graham, R.McCoy, MA., Schultz, AM., editors. Medicine I of Strategies to Improve Cardiac Arrest Survival: A Time to Act [Internet]. Washington, DC: The National Academies Press; 2015.

Available from: https://www.nap.edu/catalog/21723/strategies-to-improve-cardiac-arrest-survival-atime-to-act

3. Zhao Z-Q, Corvera JS, Halkos ME, Kerendi F, Wang N-P, Guyton RA, et al. Inhibition of myocardial injury by ischemic postconditioning during reperfusion: comparison with ischemic preconditioning. Am J Physiol Heart Circ Physiol United States. 2003 Aug; 285(2):H579-88.

4. Hausenloy DJ, Yellon DM. Ischaemic conditioning and reperfusion injury. Nat Rev Cardiol England. 2016 Apr; 13(4):193-209.

5. Segal N, Matsuura T, Caldwell E, Sarraf M, McKnite S, Zviman M, et al. Ischemic postconditioning at the initiation of cardiopulmonary resuscitation facilitates functional cardiac and cerebral recovery after prolonged untreated ventricular fibrillation. Resuscitation. 2012; 83(11):1397-403. [PubMed: 22521449]

6. Yannopoulos D, Segal N, Matsuura T, Sarraf M, Thorsgard M, Caldwell E, et al. Ischemic postconditioning and vasodilator therapy during standard cardiopulmonary resuscitation to reduce cardiac and brain injury after prolonged untreated ventricular fibrillation. Resuscitation. 2013; 84(8):1143-9. [PubMed: 23376583]

7. Bartos JA, Matsuura TR, Sarraf M, Youngquist ST, McKnite SH, Rees JN, et al. Bundled postconditioning therapies improve hemodynamics and neurologic recovery after $17 \mathrm{~min}$ of untreated cardiac arrest. Resuscitation. 2015; 87:7-13. [PubMed: 25447036]

8. Heusch G. Molecular basis of cardioprotection: signal transduction in ischemic pre-, post-, and remote conditioning. Circ Res United States. 2015 Feb; 116(4):674-99.

9. Penna C, Perrelli M-G, Pagliaro P. Mitochondrial pathways, permeability transition pore, and redox signaling in cardioprotection: therapeutic implications. Antioxid Redox Signal United States. 2013 Feb; 18(5):556-99.

10. Bartos JA, Debaty G, Matsuura T, Yannopoulos D. Post-conditioning to improve cardiopulmonary resuscitation. Curr Opin Crit Care. 2014; 20(3):242-9. [PubMed: 24751810]

11. Council, NR. Guide for the Care and Use of Laboratory Animals: Eighth Edition [Internet]. Washington, DC: The National Academies Press; 2011. Available from: https://www.nap.edu/ catalog/12910/guide-for-the-care-and-use-of-laboratory-animals-eighth

12. Riess ML, Matsuura TR, Bartos JA, Bienengraeber M, Aldakkak M, McKnite SH, et al. Anaesthetic Postconditioning at the Initiation of CPR Improves Myocardial and Mitochondrial 
Function in a Pig Model of Prolonged Untreated Ventricular Fibrillation. Resuscitation. 2014; 85(12):1745-51. [PubMed: 25281906]

13. Kristian T. Isolation of mitochondria from the CNS. Curr Protoc Neurosci United States. 2010 Jul. Chapter 7: Unit 7.22.

14. Camara, AKS., Lesnefsky, EJ., Stowe, DF. Potential Therapeutic Benefits of Strategies Directed to Mitochondria. Antioxid Redox Signal [Internet]. Vol. 13. 140 Huguenot Street, 3rd FloorNew Rochelle, NY 10801USA: Mary Ann Liebert, Inc; 2010 Aug 1. p. 279-347.Available from: http:// www.ncbi.nlm.nih.gov/pmc/articles/PMC2936955/

15. Chouchani ET, Pell VR, Gaude E, Aksentijevi D, Sundier SY, Robb EL, et al. Ischaemic accumulation of succinate controls reperfusion injury through mitochondrial ROS. Nature [Internet]. 2014 Nov 20; 515(7527):431-5. Available from: http://www.ncbi.nlm.nih.gov/pmc/ articles/PMC4255242/.

16. Gazmuri RJ, Radhakrishnan J. Protecting mitochondrial bioenergetic function during resuscitation from cardiac arrest. Crit Care Clin United States. 2012 Apr; 28(2):245-70.

17. Neumar RW, Brown CG, Van Ligten P, Hoekstra J, Altschuld RA, Baker P. Estimation of myocardial ischemic injury during ventricular fibrillation with total circulatory arrest using highenergy phosphates and lactate as metabolic markers. Ann Emerg Med UNITED STATES. 1991 Mar; 20(3):222-9.

18. Aldakkak M, Stowe DF, Lesnefsky EJ, Heisner JS, Chen Q, Camara AKS. Modulation of mitochondrial bioenergetics in the isolated Guinea pig beating heart by potassium and lidocaine cardioplegia: implications for cardioprotection. J Cardiovasc Pharmacol United States. 2009 Oct; 54(4):298-309.

19. Hurst S, Hoek J, Sheu SS. Mitochondrial Ca2+ and regulation of the permeability transition pore. J Bioenerg Biomembr. 2016 Aug.

20. Lim SY, Davidson SM, Hausenloy DJ, Yellon DM. Preconditioning and postconditioning: the essential role of the mitochondrial permeability transition pore. Cardiovasc Res Netherlands. 2007 Aug; 75(3):530-5.

21. Marcu R, Neeley CK, Karamanlidis G, Hawkins BJ. Multi-parameter measurement of the permeability transition pore opening in isolated mouse heart mitochondria. J Vis Exp United States. 2012; (67)

22. Brookes PS, Yoon Y, Robotham JL, Anders MW, Sheu S-S. Calcium, ATP, and ROS: a mitochondrial love-hate triangle. Am J Physiol Cell Physiol United States. 2004 Oct; 287(4):C817-33.

23. Tajeddine N. How do reactive oxygen species and calcium trigger mitochondrial membrane permeabilisation? Biochim Biophys Acta Netherlands. 2016 Jun; 1860(6):1079-88.

24. Gadicherla AK, Stowe DF, Antholine WE, Yang M, Camara AKS. Damage to mitochondrial complex I during cardiac ischemia reperfusion injury is reduced indirectly by anti-anginal drug ranolazine. Biochim Biophys Acta Netherlands. 2012 Mar; 1817(3):419-29.

25. Angelos MG, Kutala VK, Torres CA, He G, Stoner JD, Mohammad M, et al. Hypoxic reperfusion of the ischemic heart and oxygen radical generation. Am J Physiol Heart Circ Physiol United States. 2006 Jan; 290(1):H341-7.

26. Touboul C, Angoulvant D, Mewton N, Ivanes F, Muntean D, Prunier F, et al. Ischaemic postconditioning reduces infarct size: systematic review and meta-analysis of randomized controlled trials. Arch Cardiovasc Dis Netherlands. 2015 Jan; 108(1):39-49.

27. Sharma V, Bell RM, Yellon DM. Targeting reperfusion injury in acute myocardial infarction: a review of reperfusion injury pharmacotherapy. Expert Opin Pharmacother England. 2012 Jun; 13(8):1153-75.

28. Coute RA, Mader TJ, Sherman LD. Outcomes by rescue shock number during the metabolic phase of porcine ventricular fibrillation resuscitation. Am J Emerg Med United States. 2014 Jun; 32(6): 586-91.

29. Yeh ST, Lee H-L, Aune SE, Chen C-L, Chen Y-R, Angelos MG. Preservation of mitochondrial function with cardiopulmonary resuscitation in prolonged cardiac arrest in rats. J Mol Cell Cardiol England. 2009 Dec; 47(6):789-97. 
30. Bevers MB, Neumar RW. Mechanistic role of calpains in postischemic neurodegeneration. J Cereb Blood Flow Metab United States. 2008 Apr; 28(4):655-73.

31. Kristian T. Metabolic stages, mitochondria and calcium in hypoxic/ischemic brain damage. Cell Calcium Scotland. 2004; 36(3-4):221-33.

32. Lai TW, Zhang S, Wang YT. Excitotoxicity and stroke: identifying novel targets for neuroprotection. Prog Neurobiol England. 2014 Apr.115:157-88.

33. Zhao H. Ischemic postconditioning as a novel avenue to protect against brain injury after stroke. $\mathbf{J}$ Cereb Blood Flow Metab [Internet]. 2009 May 25; 29(5):873-85. Available from: http:// www.ncbi.nlm.nih.gov/pmc/articles/PMC2736291/.

34. Xing B, Chen H, Zhang M, Zhao D, Jiang R, Liu X, et al. Ischemic postconditioning inhibits apoptosis after focal cerebral ischemia/reperfusion injury in the rat. Stroke United States. 2008 Aug; 39(8):2362-9.

35. Kilbaugh, TJ., Sutton, RM., Karlsson, M., Hansson, MJ., Naim, MY., Morgan, RW., et al. J Am Hear Assoc Cardiovasc Cerebrovasc Dis [Internet]. Vol. 4. Chichester, UK: John Wiley \& Sons, Ltd; 2015 Sep 14. Persistently Altered Brain Mitochondrial Bioenergetics After Apparently Successful Resuscitation From Cardiac Arrest; p. e002232Available from: http:// www.ncbi.nlm.nih.gov/pmc/articles/PMC4599507/

36. Reynolds JC, Salcido DD, Menegazzi JJ. Coronary Perfusion Pressure and Return of Spontaneous Circulation after Prolonged Cardiac Arrest. Prehosp Emerg Care [Internet]. 2010; 14(1):78-84. Available from: http://www.ncbi.nlm.nih.gov/pmc/articles/PMC2922866/.

37. Schwartz BG, Kloner RA. Coronary no reflow. J Mol Cell Cardiol England. 2012 Apr; 52(4):87382.

38. Buchholz, B., Donato, M., D’Annunzio, V., Gelpi, RJ. Mol Cell Biochem. Vol. 392. Springer; 2014. Ischemic postconditioning: mechanisms, comorbidities, and clinical application; p. 1-12. 
a)

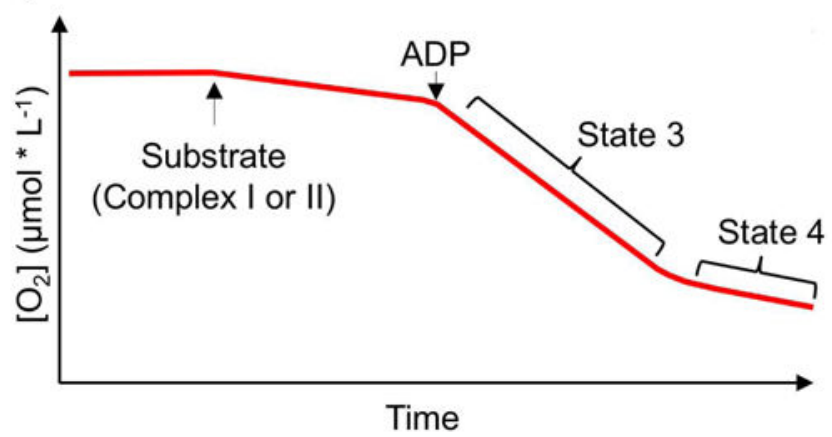

b)

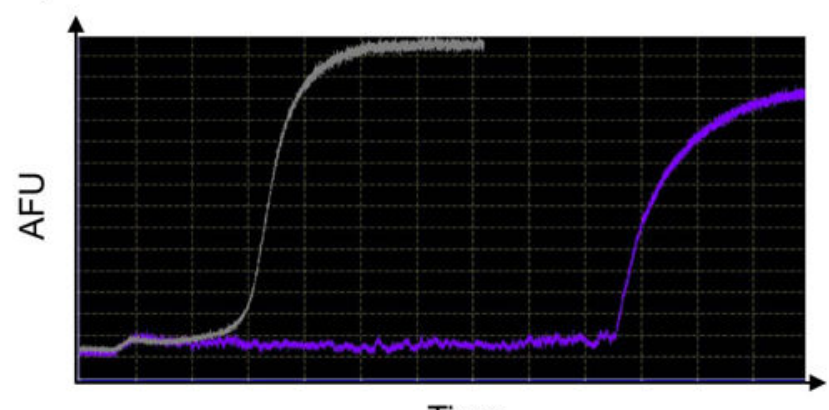

Time c)

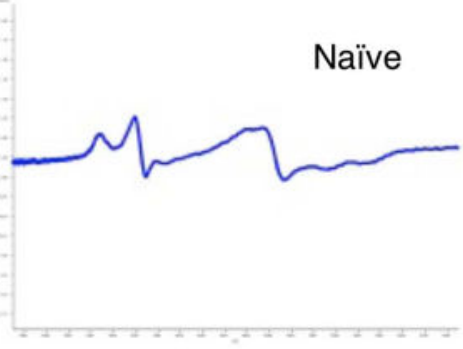

e)

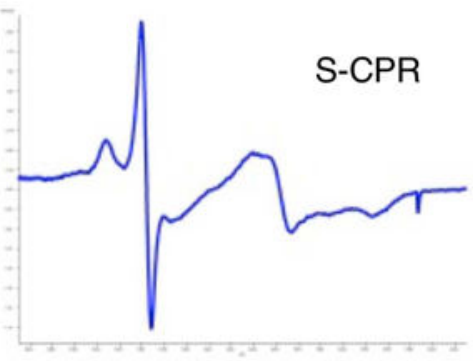

d)

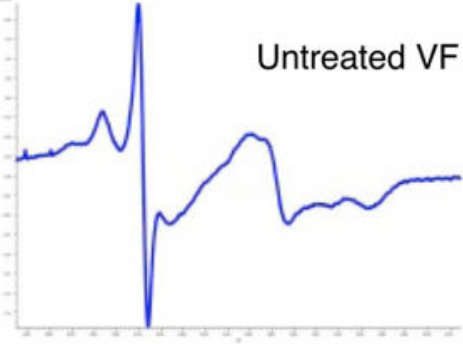

f)

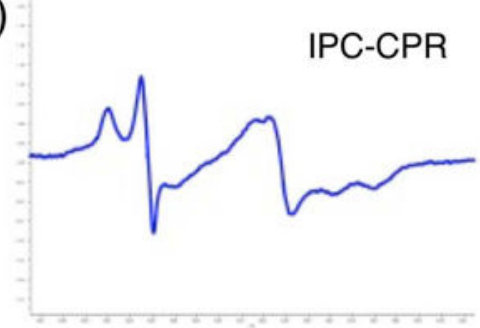

g)

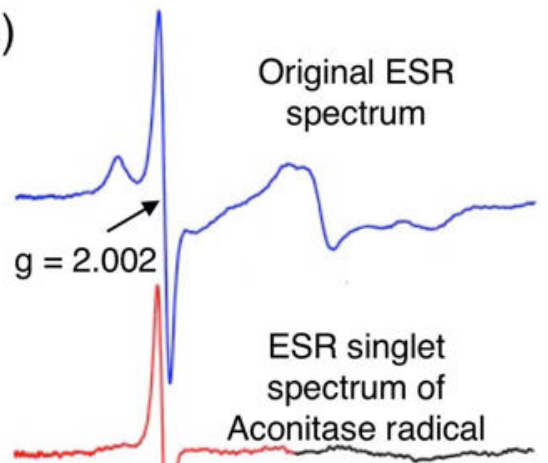

Tissue background bioradicals

Figure 1.

Experimental Design. Animals were randomized to receive no ischemia (Naïve) or cardiac arrest by ventricular fibrillation (VF), and cardiac arrest groups were further randomized to receive no CPR (Untreated VF), standard cardiopulmonary resuscitation (S-CPR), or ischemic postconditioning at the initiation of CPR (IPC-CPR). After surgical preparation in Naïve or 19 min after induction of VF in ischemic groups, the heart or brain was harvested for isolation of mitochondria via differential centrifugation. 

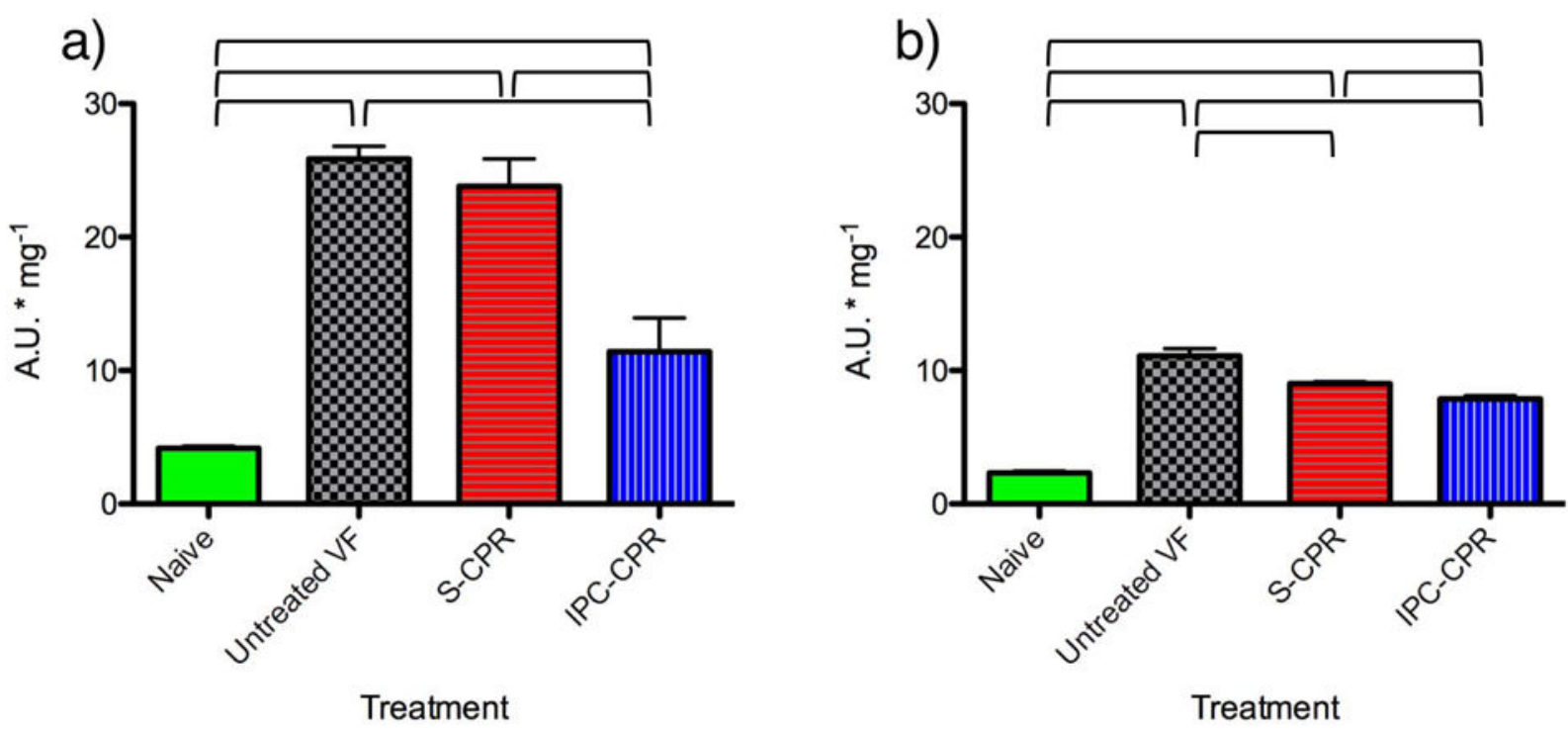

Figure 2.

Coronary perfusion pressure (CPP) during cardiopulmonary resuscitation (CPR) with and without ischemic postconditioning (IPC). IPC was achieved via 3 cycles of 20-seconds compressions followed by 20 seconds pause during the first 2 minutes of CPR. IPC-CPR increased CPP compared to S-CPR following an intravenous bolus of epinephrine $(0.125$ $\mu \mathrm{g} / \mathrm{kg}$ ) administered at the start of the 4th minute of CPR (a). The 4-minute CPP average did not differ between groups (b). Data are shown as mean \pm SEM. Data from the same time point were compared across treatment with unpaired t-test, * indicates $\mathrm{p}<0.05$. 
Complex I

a)

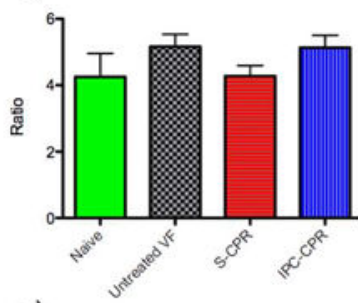

c)

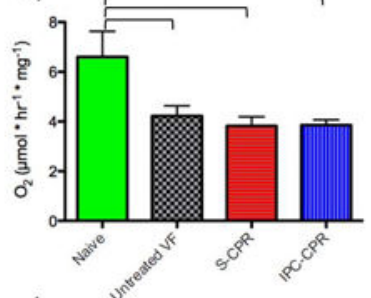

e)

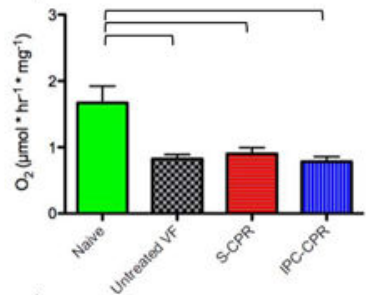

g)
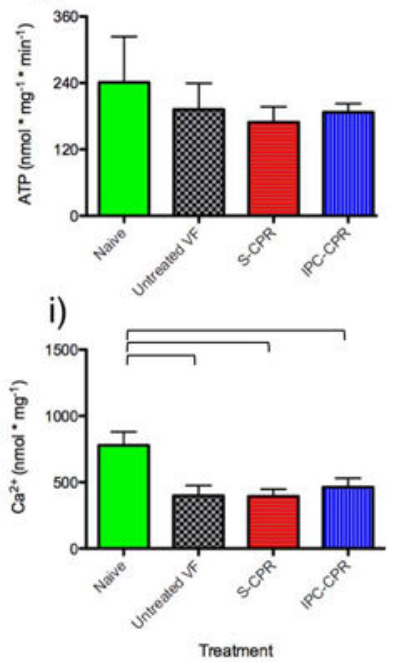

b)

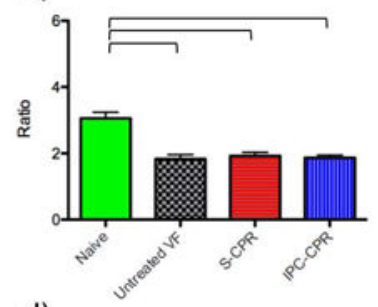

d)

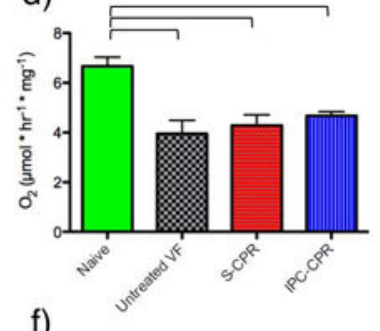

f)

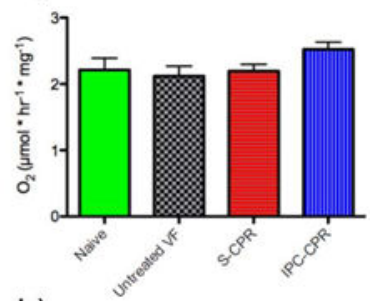

h)
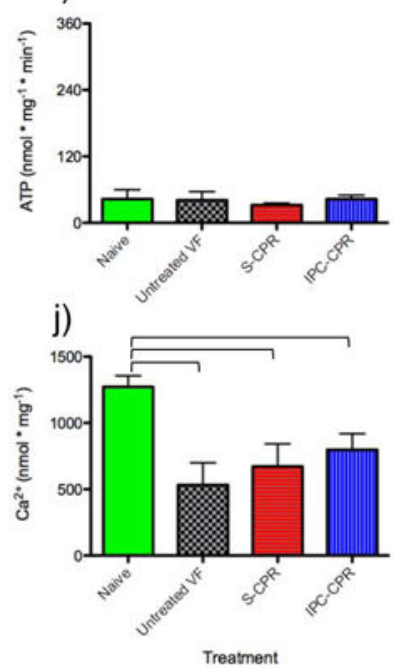

Figure 3.

Cardiac mitochondrial function tests. Function tests were assessed with complex I substrates pyruvate and malate (left column) or complex II substrate succinate plus complex I inhibitor rotenone (right column). RCI (a, b) was calculated as the ratio of State 3 (S3; c, d) to State 4 (S4; e, f) respiration. RCI decreased during Untreated VF compared to Naïve primarily due to increased S4 respiration. IPC-CPR increased RCI compared to Untreated VF and S-CPR mediated by increased S3 and decreased S4 respiration. The rate of ATP synthesis decreased during Untreated VF compared to Naïve and was recovered with IPC-CPR (g, h). CRC 
increased with IPC-CPR compared to Untreated VF in the presence of complex I substrates (i), whereas CRC decreased in all ischemic groups compared to Naïve in the presence of complex II substrates (j). Data are shown as mean \pm SEM brackets indicate $p<0.05$ between groups. 
Complex I

a)

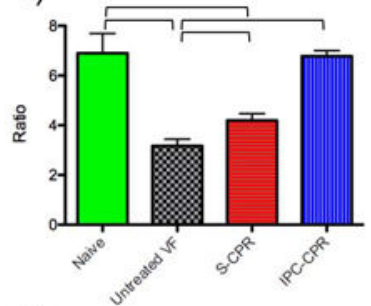

c)

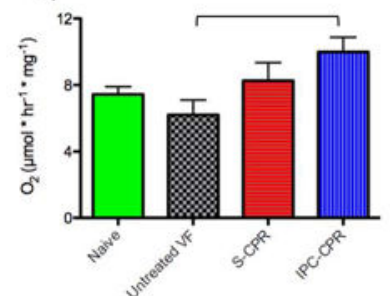

e)

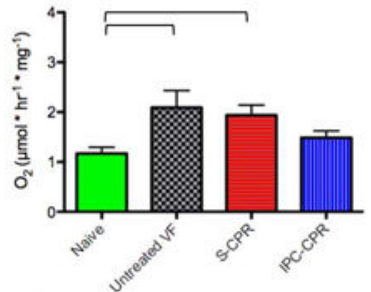

g)
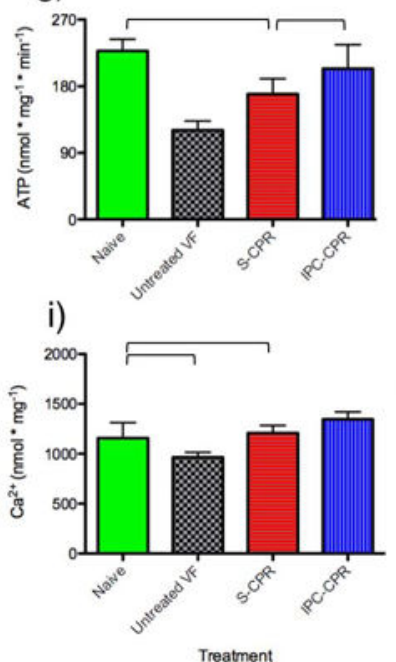

Complex II

b)

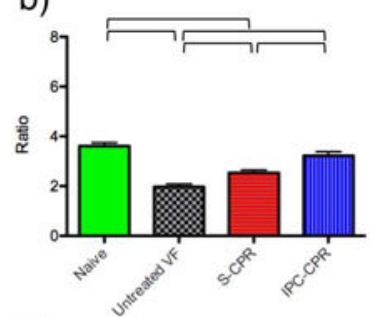

d)

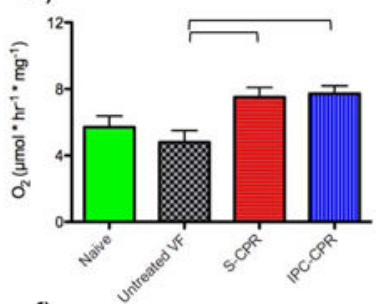

f)

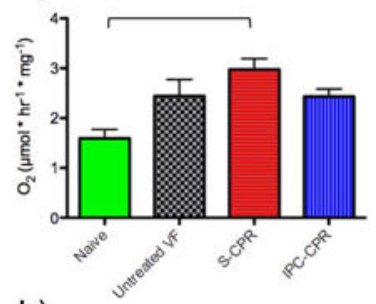

h)
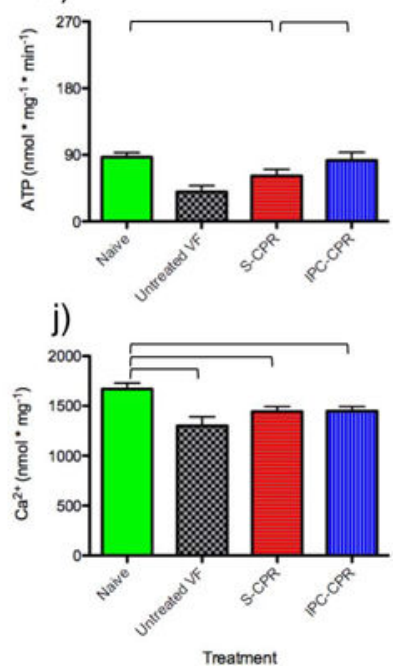

Figure 4.

Cerebral mitochondrial function tests. Function tests were assessed with complex I substrates pyruvate and malate (left column) or complex II substrate succinate plus complex I inhibitor rotenone (right column). RCI (a, b) was calculated as the ratio of State 3 (S3; c, d) to State 4 (S4; e, f) respiration. Complex I RCI was unaffected by treatment due to proportional decreases in S3 and S4 respiration in all ischemic groups. Complex II RCI decreased in all ischemic groups compared to Naïve due to decreased $\mathrm{S} 3$ respiration. The rate of ATP synthesis was unchanged between treatments $(\mathrm{g}, \mathrm{h})$. CRC decreased in all 
ischemic groups compared to Naïve ( $i, j)$. Data are shown as mean \pm SEM; brackets indicate $\mathrm{p}<0.05$ between groups. 


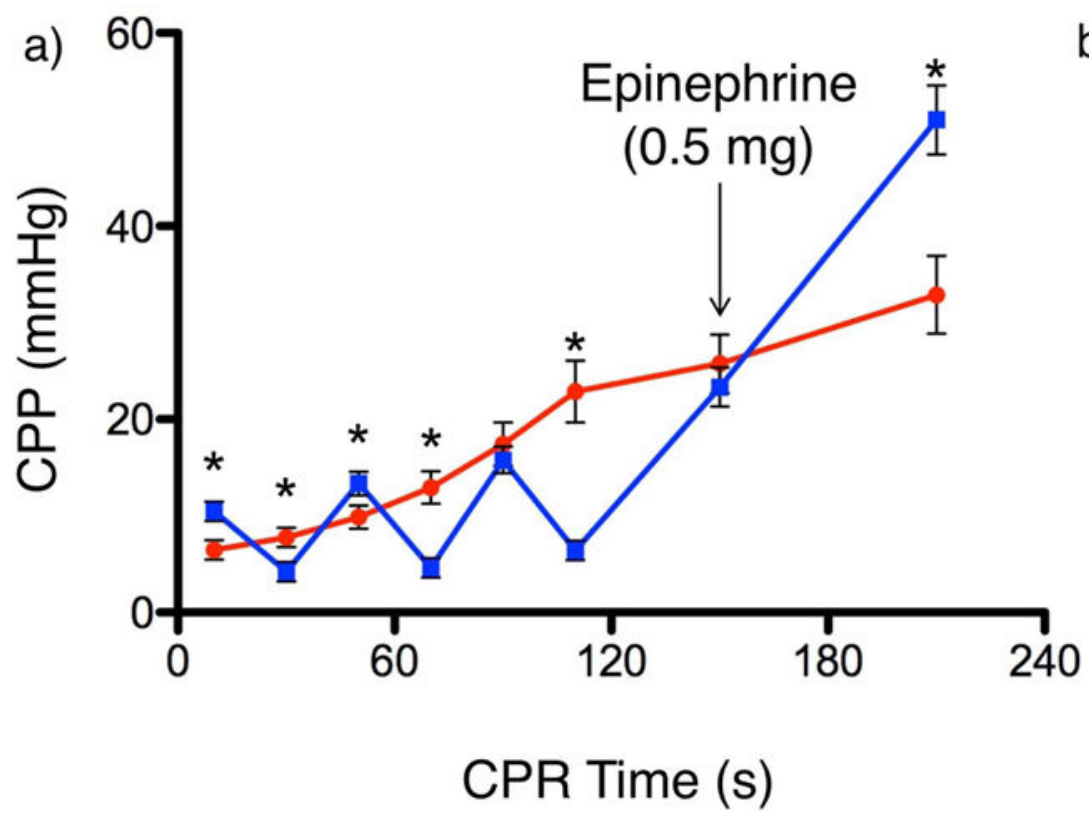

b)

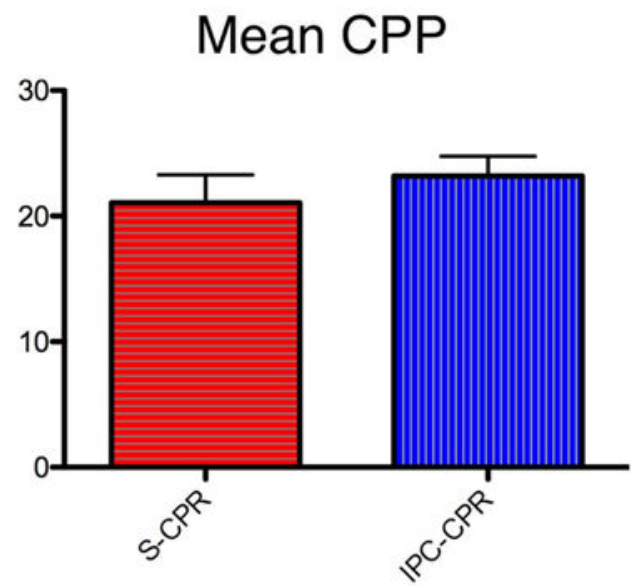

Figure 5.

ESR spectra from fresh-frozen heart and brain tissue. Aconitase radical intensity for cardiac (a) and brain (b) samples. Untreated VF increased oxidized aconitase compared to Naïve treatment. Postconditioning with IPC-CPR decreased aconitase ROS production from cardiac and brain biopsies taken during the 4 th minute of CPR compared to S-CPR. Data are shown as mean \pm SEM; brackets indicate $\mathrm{p}<0.05$ between groups. 


\section{Table 1}

Baseline hemodynamics and $\mathrm{ABG}$ values prior to ischemia.

Baseline parameters. There were no differences in hemodynamic or ABG values between treatment groups prior to ischemia. MAP, mean arterial pressure; RAP, right atrial pressure. Data are shown as mean $\pm \mathrm{SEM}$.

\begin{tabular}{lcccc}
\hline & \multicolumn{4}{c}{ Treatment Group } \\
Parameter & Naïve & Untreated VF & S-CPR & IPC-CPR \\
\hline $\mathrm{MAP}(\mathrm{mmHg})$ & $80.7 \pm 3.7$ & $87.1 \pm 6.3$ & $86.7 \pm 3.7$ & $88.7 \pm 4.7$ \\
$\mathrm{RA}(\mathrm{mmHg})$ & $6.5 \pm 0.6$ & $6.0 \pm 0.7$ & $5.9 \pm 0.7$ & $7.0 \pm 0.7$ \\
$\mathrm{pH}$ & $7.46 \pm 0.01$ & $7.47 \pm 0.01$ & $7.47 \pm 0.01$ & $7.46 \pm 0.01$ \\
$\mathrm{pCO}(\mathrm{mmHg})$ & $42.7 \pm 0.8$ & $41.0 \pm 1.1$ & $40.3 \pm 0.7$ & $40.5 \pm 0.4$ \\
$\mathrm{pO}_{2}(\mathrm{mmHg})$ & $96.0 \pm 3.8$ & $87.9 \pm 4.3$ & $92.9 \pm 3.0$ & $97.9 \pm 4.0$ \\
$\mathrm{Temp}\left({ }^{\circ} \mathrm{C}\right)$ & $37.5 \pm 0.4$ & $37.2 \pm 0.5$ & $37.8 \pm 0.2$ & $37.7 \pm 0.2$ \\
Weight $(\mathrm{kg})$ & $41.8 \pm 0.7$ & $42.9 \pm 1.1$ & $41.6 \pm 0.6$ & $42.5 \pm 0.7$ \\
Number & 12 & 13 & 19 & 19 \\
\hline
\end{tabular}

